\title{
Primeros registros de leucismo en la ardilla de nuca blanca Simosciurus nebouxii (Rodentia: Sciuridae) en la ciudad de Lima, Perú
}

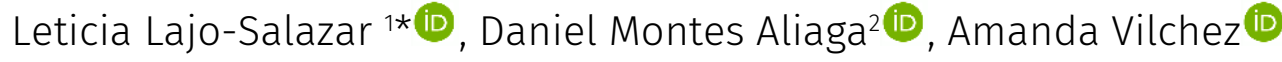 \\ 1 Centro de Ornitología y Biodiversidad (CORBIDI). Santa Rita No. 105 Of. 202, Urb. Huertos de San Antonio, Surco, Lima, \\ Perú. \\ 2 FaunaVet-PERÚ. Jirón Sánchez Cerro №16, Ate, Lima, Perú. \\ * Correspondencia: Leticia.lajo@gmail.com
}

\section{Resumen}

El leucismo es una anomalía cromática que se presenta en una amplia variedad de mamíferos y otros vertebrados, aunque de manera poco frecuente. Los reportes de ardillas arborícolas con esta condición se han hecho en especies de Asia, Norteamérica y Europa, pero para especies de ardillas neotropicales son casi inexistentes. Esta nota reporta la ocurrencia de leucismo en ardillas de nuca blanca que habitan las áreas verdes de tres distritos de la ciudad de Lima, siendo necesarios futuros estudios genéticos y poblacionales para determinar su causa.

Palabras clave: albinismo, anomalía cromática, fauna urbana, Sciurus

Abstract

Leucism is a color abnormality that occurs in a wide variety of mammals and other vertebrates, although it is infrequent. Reports of tree squirrels with this condition have been made in species from Asia, North America, and Europe, but for Neotropical squirrel species, they are almost nonexistent. This note reports the occurrence of leucism in white-naped squirrels that inhabit the green areas of three districts of the city of Lima. Future genetic and population studies are needed to determine the cause.

Key words: albinism, chromatic abnormality, Sciurus, urban fauna

La melanina en sus dos formas, eumelanina y feomelanina, es el pigmento responsable del color en la piel y pelo de los mamíferos (Slominski et al. 2004; Lamoreaux et al. 2010). Tanto en estos como en otros vertebrados se pueden producir deficiencias para producir o depositar melanina en los tejidos, provocando fenotipos distintos (Van Grouw 2006; Lamoreaux et al. 2010; Abreu et al. 2013) conocidos como leucismo y albinismo. Se denomina leucismo a la ausencia parcial de melanina en el tegumento, originando individuos de apariencia blanca o amarillenta, pero conservando coloración oscura en ojos 
y/o extremidades, mientras que el término albinismo se reserva para los individuos con ausencia total de pigmentos en los tejidos dando además una apariencia rojiza a los ojos al transparentarse los vasos sanguíneos bajo los tejidos oculares (Van Grouw 2006, Abreu et al. 2013). En ardillas arborícolas la ocurrencia de albinismo y leucismo se ha presentado usualmente a partir de reportes de colecta u observaciones de unos pocos individuos cada vez, con sus excepciones (Tabla 1). La gran mayoría de reportes en la literatura científica se refieren a especies de Asia y Norteamérica, por lo que el registro que se presenta en esta nota constituye uno de los primeros para una especie de la región Neotropical.

TABLA 1. Listado de registro de ardillas arborícolas albinas (A) y leucísticas, total (L) o parcial (LP). El símbolo (?) indica registros de ardillas de pelaje blanco de las que no se discierne condición específica.

\begin{tabular}{|c|c|c|c|c|}
\hline Subfamilia/Especie & Condición & Provincia, País & Individuos & Referencia \\
\hline \multicolumn{5}{|l|}{ CALLOSCIURINAE } \\
\hline Callosciurus pygerythrus & A & Assam, India & 1 & Kalita 2009 \\
\hline Funambulus palmarum & $\mathrm{L}$ & Tamil Nadu, India & 1 & Samson et al. 2017 \\
\hline \multirow[t]{6}{*}{ Funambulus pennantii } & $A$ & Chandigarh, India & 1 & Chaturvedi \& Ghose 1984 \\
\hline & A & Rajastán, India & 1 & Sharma 2004 \\
\hline & $A$ & Maharashtra, India & 2 & Mahabal et al. 2005 \\
\hline & A & Rajastán, India & 1 & Mehara et al. 2007 \\
\hline & $\mathrm{L}$ & Ittar Pradesh, India & 1 & Ramesh et al. 2019 \\
\hline & $\mathrm{L}$ & Uttarakhand, India & 1 & Kamalakannan et al. 2019 \\
\hline Funambulus tristriatus & $A / L P$ & Goa, India & $2 / 1$ & Sayeed et al. 2015 \\
\hline \multicolumn{5}{|l|}{ RATUFINAE } \\
\hline Ratufa indica & A & Maharashtra, India & 1 & Sayeed et al. 2015 \\
\hline \multicolumn{5}{|l|}{ SCIURINAE } \\
\hline Guerlinguetus brasiliensis & $\mathrm{L}$ & Río de Janeiro, Brasil & 2 & Tavares et al 2019 \\
\hline Notosciurus granatensis & LP & El Oro, Ecuador & 1 & Ramirez-Jaramillo 2019 \\
\hline Sciurus alberti & $?$ & Arizona, EUA & 1 & Keith 1965 \\
\hline \multirow[t]{4}{*}{ Sciurus carolinensis } & $A$ & - & - & Dunn 1921 \\
\hline & A & Illinois, EUA & $106-182$ & Stencel \& Ghent 1987 \\
\hline & A & Reino Unido & 3 & Esteve \& Jeffery 1998 \\
\hline & $\mathrm{L}$ & Texas, EUA & 4 & McCardle 2012 \\
\hline Sciurus niger & $\mathrm{L}$ & Texas, EUA & 2 & McCardle 2012 \\
\hline \multirow{2}{*}{ Sciurus vulgaris } & $? / L P$ & - & - & Jones 1923 \\
\hline & LP & Overijssel, Holanda & 1 & Hoekstra 2004 \\
\hline \multirow[t]{11}{*}{ Tamiasciurus hudsonicus } & $A$ & Nueva York, EUA & $\begin{array}{l}\text { "pequeña } \\
\text { población" }\end{array}$ & Mearns 1898 \\
\hline & $?$ & Noroeste, Canada & 1 & McFarlane 1905 \\
\hline & $?$ & Nueva York, EUA & 2 & Seton 1909 \\
\hline & A & Michigan, EUA & 2 & Dunn 1921 \\
\hline & $A / ? / L P$ & Michigan, EUA & 1/"varias"/1 & Wood 1922 \\
\hline & $?$ & Michigan, EUA & 1 & Dice 1925 \\
\hline & A & Indiana, EUA & 2 & Engles 1933 \\
\hline & LP & Nueva York, EUA & 4 & Layne 1954 \\
\hline & $A$ & Alberta, Canada & 1 & Wood 1965 \\
\hline & $\mathrm{L}$ & Yucón, Canada & 1 & Jung \& Slough 2012 \\
\hline & A & Quebec, Canada & 1 & Ferron \& Laplante 2013 \\
\hline
\end{tabular}

La Ardilla de nuca blanca Simosciurus nebouxii Geoffroy St.-Hilaire (1855) es una especie común del bosque estacionalmente seco ubicado al norte del Perú y extremo sur de Ecuador (Emmons \& Feer 1997; Tirira 2007; De Vivo \& Carmignoto 2015). Fue introducida en Lima hacia fines de la década del 70, y ahora es común en varios distritos de la ciudad y en algunas zonas suburbanas (Jessen et al. 2010). El color normal de su pelaje (Figura 1a) 
es gris claro, con las partes posteriores del dorso y las patas traseras de color amarillo pálido o anaranjado claro, la cabeza del mismo color que el cuerpo, las partes distales de las patas son de color marrón oscuro o negro, el vientre es amarillo jaspeado con gris, la cola con pelos negros en la base y gris claro en las puntas a excepción de la sección proximal que es amarillenta o anaranjado claro (Emmons \& Feer 1997; Eisenberg \& Redford 1999; De Vivo \& Carmignotto 2015). Aunque pueden observarse variaciones en la intensidad de la coloración del pelaje (Figuras 1 b y $1 c$ ), en la literatura no se tienen registros de anomalías marcadas a excepción del individuo melánico mencionado por Eisenberg \& Redford (1999) bajo la denominación de "black morph".

Los primeros registros no publicados de avistamientos de S. nebouxii con anomalías en el color de pelaje en la ciudad de Lima fueron en el año 2008. Uno de ellos por Sarah LandeoYauri, durante su investigación sobre el comportamiento de la ardilla de nuca blanca en Lima; obteniendo una fotografia borrosa de un individuo de pelaje amarillento en el Lima Golf Club, San Isidro. El otro se trató de un individuo albino (con ojos rojos) observado por Evelyn Tavera Fernández durante una evaluación de aves en áreas verdes de la ciudad. Ambos eventos se dieron en el distrito de San Isidro y no se tuvo conocimiento de otras ocurrencias. A finales del año 2017 se supo de avistamientos de "ardillas blancas" en diferentes distritos de la ciudad de Lima. Por ello, se hizo una recopilación de evidencias fotográficas y, entre el mes de setiembre de 2017 y marzo de 2018, se realizaron visitas a las áreas donde se reportaron los avistamientos y zonas aledañas en busca de los individuos para confirmar su existencia El sexo y edad de las ardillas encontradas se determinó según lo descrito por Allen (1943).

Encontramos tres individuos leucísticos hembras, todos. El primero se trató de una hembra adulta hallada en una de las áreas verdes del campus de la Universidad Nacional Agraria La Molina, distrito de La Molina (Figura 1d). Dicho individuo exhibía pelaje blanco amarillento, a excepción de la parte posterior del dorso y las patas traseras, que eran anaranjado pálido; y la sección distal de las cuatro patas, de color gris claro. La nuca estaba cubierta de pelo blanco, como los individuos de coloración típica. Los ojos eran de color marrón oscuro, descartando un caso de albinismo. Se pudo confirmar que el individuo habitaba un área relativamente aislada del campus con densa vegetación arbórea y arbustiva. A la fecha se sabe que continúan avistamientos ocasionales de individuos con estas características en el área y zonas aledañas dentro del campus.

Otro individuo de similares características fue registrado en el Zoológico Parque de las Leyendas, distrito de San Miguel, donde hay una población silvestre. Se trataba de una hembra que pudo ser observada en varias oportunidades (Figura 1e), con las mismas características del primer individuo a excepción del color de la parte distal de las patas, que en este caso se presenta ligeramente anaranjado. En todos los casos en los que se pudo observar a este individuo se encontraba dentro del recinto de los canguros que forman parte de la colección del zoológico, alimentándose de los vegetales contenidos en la comida ofrecida a estos. Al igual que en el primer caso, los avistamientos continúan a la fecha, sin haberse podido confirmar que se trate del mismo individuo.

El tercer individuo fue una hembra adulta registrada en la Residencial San Felipe, distrito de Jesús María, el cual está compuesto de edificios de hasta 15 pisos, pero cuenta con numerosas áreas verdes. El registro se hizo con la cámara de un celular (Figuras 1f y 1g) por un transeúnte que frecuentaba la zona, posteriormente su presencia fue confirmada durante nuestras búsquedas en el área, aunque no se pudo obtener una fotografia más clara. En este caso el pelaje tenía una coloración blanquecina más uniforme debido a la 
ausencia de pelos de tinte anaranjado en la parte posterior del dorso o las extremidades anteriores. El parche blanco en la nuca, por ello, era menos notorio. El color de los ojos también era oscuro. La zona en la que se pudo observar se trata de un área verde con árboles de distintas alturas y escasos arbustos en la que domina la cobertura de césped.

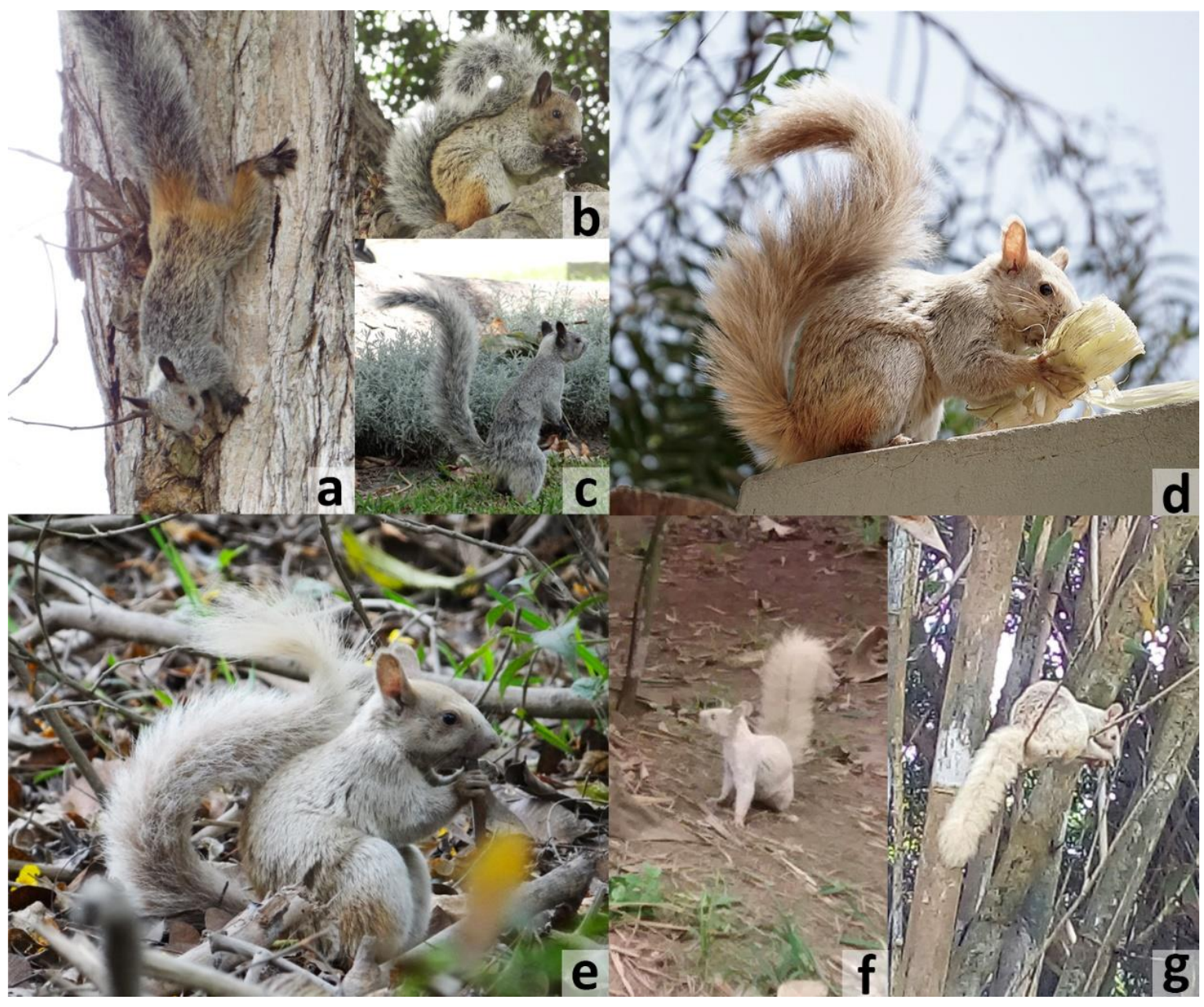

FIGURA 1. Individuos de S. nebouxii: (a) con coloración típica, (b y c) con variaciones comunes, y leucísticos reportados en los distritos de (d) La Molina, (e) San Miguel y (f y g) Jesús María.

Las anomalías cromáticas en vertebrados, en general, se deben a cambios a nivel genético a consecuencia de la contaminación y/u otras alteraciones en el ambiente (Mфller \& Mousseau 2001), deriva genética (Hafner \& Hafner 1987), selección natural (Schneider et al. 2015) o endogamia (Holyoak 1978; Bensch et al. 2000; Łopucki \& Mróz 2010). Sin embargo, el leucismo en particular ha sido asociado también con otras causas, como una baja calidad de nutrientes en la dieta (Rollin 1959; Short \& Laybourn 1967) o daño en los folículos pilosos (Phillips 1954). Cualquiera de estas causas puede darse en ambientes urbanos debido a la interaccion de los múltiples factores que se presentan en estos espacios, como lo describen Izquierdo et al. (2018). Asimismo, ya que estos son los primeros reportes de leucismo en Simosciurus nebouxii y que se trata de una especie muy poco estudiada, son necesarios análisis poblacionales, ecológicos y genéticos para entender la ocurrencia de esta anomalía en la población de ardillas de nuca blanca que habitan la ciudad de Lima, así como evaluar si existen registros de leucismos en las poblaciones nativas al norte del Perú y sur de Ecuador. 


\section{AGRADECIMIENTOS}

A Sarah Landeo-Yauri y Evelyn Tavera Fernández por la información de avistamientos de 2008 y a Rodrigo Sánchez Ludeña por brindar la fotografia y la información del individuo reportado en Jesús María.

\section{REFERENCIAS}

Abreu MSL, Machado R, Barbieru F, Freitas NS, Oliveira LR. 2013. Anomalous colour in Neotropical mammals: a review with new records for Didelphis sp. (Didelphidae, Didelphimorphia) and Arctocephalus australis (Otariidae, Carnivora). Brazilian Journal of Biology 73: 185-194. https:// doi.org/10.1590/S1519-69842013000100020

Allen DL. 1943. Michigan fox squirrel management. Michigan, USA. Michigan Department of Conservation, Game Division.

Bensch S, Hansson B, Hasselquist D, Nielsen B. 2000. Partial albinism in a semi-isolated population of Great Reed Warblers. Hereditas 133:167-170. http:// doi.org/10.1111/j.1601-5223.2000.t011-00167.x

Chaturvedi Y, Ghose AK. 1984. A case of albinism in the five-striped palm squirrel Funambulus pennantil Wroughton. Bulletin of the Zoological Survey of India 6(1-3): 321-322.

De Vivo M, Carmignotto AP. 2015. Family Sciuridae G. Fisher, 1817. In: Patton JL., Pardiñas UFJ, D’Elia G, editors. Mammals of South America, Volume 2: Rodents. Chicago, USA: University of Chicago Press. p. 1-48.

Dice LR. 1925. A survey of the mammals of Charlevoix County, Michigan, and vicinity. University of Michigan, Occasional Papers of the Museum of Zoology 159: 1-33.

Dunn LC. 1921. Unit character variation in rodents. Journal of Mammalogy 2: 125-140. https://doi.org/10.2307/1373601

Eisenberg JF, Redford KH. 1999. Mammals of the neotropics, Volume 3, the Central Neotropics: Ecuador, Peru, Bolivia, Brazil. Chicago, USA: University of Chicago Press.

Emmons L, Feer F. 1997. Neotropical rainforest mammals: a field guide (Second Edition). Chicago, USA: University of Chicago Press.

Engles WL. 1933. Notes on the mammals of St. Joseph County, Indiana. The American Midland Naturalist 14(1): 1-16. https:// doi.org/10.2307/2420047

Esteve J, Jeffery G. 1998. Reduced retinal deficits in the albino mammal with cone rich retina: a study of the ganglion cell layer at the area centralis of the pigmented and albino grey squirrels. Vision Research 38(6): 937-940. https:// doi.org/10.1016/s0042-6989(97)00229-0

Ferron J, Laplante P. 2013. Report of an albino red squirrel from Sainte-Luce, Quebec, Canada. Northeastern Naturalist 20(4): N16-N18. https:/ / doi.org/10.1656/045.020.0411

Hafner MS, Hafner DJ. 1987. Geographic distribution of two Costa Rican species of Orthogeomys, with comments on dorsal pelage markings in the Geomyidae. The Southwestern Naturalist 32(1): 5-11. http:// doi.org/10.2307/3672003

Hoekstra B. 2004. Een geval van partieel-albinisme bij de eekhoorn (Sciurus vulgaris Linnaeus, 1758). Lutra 47: 47-50.

Holyoak DT. 1978. Variable albinism of the flight feathers as an adaptation of recognition of individual birds in some Polynesian populations of Acrocephalus warblers. Ardea 66:112117. 
Izquierdo L, Thomson RL, Aguirre JI, Díez-Fernández A, Faivre B, Figuerola J, Ibáñez-Álamo JD. 2018. Factors associated with leucism in the common blackbird Turdus merula. Journal of Avian Biology 49: e01778. https://doi.org/10.1111/jav.01778

Jessen RR, Merrick MJ, Koprowski JL, Ramirez O. 2010. Presence of Guayaquil squirrels on the central coast of Peru: an apparent introduction. Mammalia 74:443-444. http:// doi.org/10.1515/MAMM.2010.057

Jones SVH. 1923. Color variations in wild animals. Journal of Mammalogy 4(3): 172-177. https:// doi.org/10.2307/1373567

Jung TS, Slough BG. 2012. Isabelline coloration in American red squirrels (Tamiasciurus hudsonicus) from the Yukon. Northwestern naturalist 93: 163-164. https:/ / doi.org/10.1898/ nwn12-07.1

Jones SVH. 1923. Color variations in wild animals. Journal of Mammalogy 4(3): 172-177. https:// doi.org/10.2307/1373567

Kalita G. 2009. Note on breeding and parental care behaviours of albino Hoary-bellied squirrel Callosciurus pygerythrus (Rodentia: Sciuridae) in Sibsagar district of Assam, India. Journal of Threatened Taxa 1(6): 358-360. https:// doi.org/10.11609/JoTT.01770.358-60

Kamalakanannan M, Chandra K, Venkatraman C. 2019. A leucistic Northern palm Squirrel from Dehradun, India. Zoo's Print Journal 34(11): 29-31.

Keith JO. 1965. The Albert squirrel and its dependence on ponderosa pine. Ecology 46(1): 150-163. https:// doi.org/10.2307/1935266

Lamoreux LM, Delmas V, Larue L, Bennett D. 2010. The Colors of Mice: A Model Genetic Network. London: Wiley-Blackwell.

Layne JN. 1954. The Biology of the Red Squirrel, Tamiasciurus hudsonicus loquax (Bangs), in Central New York. Ecological Monographs 24(3): 227-268. https:// doi.org/10.2307/1948465

Łopucki R, Mróz I. 2010. Cases of colouration anomalies in small mammals of Poland and reasons for their incidence. Annales Universitatis Mariae Curie-Skłodowska. Sectio C, Biologia 65:6776.

Mahabal A, Sharma RM, Pradhan MS. 2005. A Case of total albinism in the five striped palm squirrel Funambulus pennanti Wroughton in Sindhudurg District, Maharashtra State. The Journal of the Bombay Natural History Society 102: 98-99.

McCardle H. 2012. Albinism in wild vertebrates. Thesis, Master of Science. Texas State University San Marcos. San Marcos, Texas.

McFarlane R. 1905. Notes on mammals collected and observed in the northern Mackenzie River District, Northwest Territories of Canada, with remarks on explorers and explorations of the far north. Proceedings of the United States Natural Museum 28(1405): 673-764. https:// doi.org/10.5479/si.00963801.28-1405.673

Mearns EA. 1898. A study of the vertebrate fauna of the Hudson Highlands, with observations on the Mollusca, Crustacea, Lepidoptera, and the flora of the region. Bulletin of the American Museum of Natural History 10: 303-352.

Mehara SP, Kharwar JS, Kharwar NS. 2007. Second record of albino five striped palm squirrel Funambulus pennanti Whoughton from Udaipur, Rajasthan. The Journal of the Bombay Natural History Society 104(3): 344.

Mфller AP, Mousseau TA. 2001. Albinism and phenotype of Barn Swallows (Hirundo rustica) from Chernobyl. Evolution 55:2097-2104. https:// doi.org/10.1111/j.0014-3820.2001.tb01324.x

Phillips ARK. 1954. The cause of partial albinism in a Great tailed Grackle. The Wilson Bulletin 66: 66. 
Ramesh SY, Pinkra GP, Kerketta D, Kumar D. 2019. First Record of Leucism in Five Striped Palm Squirrel, Funambulus pennantii (Rodentia: Sciuridae) from North India. International Journal of Current Microbiology and Applied Sciences 8(10): 1956-1961. https:/ / doi.org/10.20546/ijcmas.2019.810.228

Ramirez-Jaramillo SM. 2019. Un caso de leucismo parcial en la ardilla de cola roja Notosciurus granatensis (Rodentia: Sciuridae) en el Sur-oeste de Ecuador. Mammalogy Notes 5(2): 24-25. https:// doi.org/10.47603/manovol5n2.24-25

Rollin N. 1959. White plumage in blackbirds. Bulletin of the British Ornithologists' Club 79(6): 92-96.

Samson A, Ramakrishnan B, Bargavi S. 2017. Leucism in the three-striped palm squirrel (Funambulus palmarum) at Gudalur Forest Division, Tamil Nadu, Southern India. Therya 8(3): 261-262. https://doi.org/10.12933/therya-17-503

Sayeed A, Mahabal A, Nale A. 2015. A first record of albinism in Jungle Palm Squirrels Funambulus tristriatus (Rodentia: Sciuridae) from India. Zoos' Print Journal 30(12): 18-19.

Schneider A, Henegar C, Day K, Absher D, Napolitano C, Silvera L, David VA, O'Brien SJ, MenottiRaymond M, Barch GS, Eizirik E. 2015. Recurrent evolution of melanism in South American felids. PLoS Genetics 11:e1004892. https:// doi.org/10.1371/journal.pgen.1004892

Seton ET. 1909. Life-Histories of Northern Animals: an account of the mammals of Manitoba. Vol. 1. - Grass-Eaters. New York, USA. Scribner's Sons.

Sharma SK. 2004. Occurrence of albino Common Palm Civet and Northern Palm Squirrel in southern Rajasthan. Zoos' Print Journal 19(5): 1483. https:/ / doi.org/10.11609/ JOTT.ZPJ.1096.1483

Short LL, Laybourn R. 1967. An instance of "White wing-barring" in the common crow. The Wilson Bulletin 79(1): 113-114.

Slominski A, Tobin DJ, Shibajara S, Wortsman J. 2004. Melanin pigmentation in mammalian skin and its hormonal regulation. Physiological Reviews 84: 1155-1228. https:// doi.org/10.1152/ physrev.00044.2003

Stencel JE, Ghent AW. 1987 Analyses of Annual Surveys of White and Gray Squirrels (Sciurus carolinensis) in Olney, Illinois, 1977-1986. The American Midland Naturalist 118(2): 251-257. https:// doi.org/10.2307/2425782

Tavares MS, Aguilar LS, Salvador CH, Galliez M. 2019. Beyond the color: the implications of pigmentation polymorphism in the activity behavior of a Neotropical squirrel. Mammalia 84(3): 294-298. https:// doi.org/10.1515/mammalia-2019-0025

Tirira D. 2007. Guía de campo de los mamíferos del Ecuador. Quito, Ecuador: Editorial Murciélago Blanco.

Van Grouw H. 2006. Not every white bird is an albino: sense and nonsense about colour aberrations in birds. Dutch Birding 28: 79-89.

Wood NA. 1922. The mammals of Washtenaw County, Michigan. University of Michigan, Occasional Papers of the Museum of Zoology 123: 1-23.

Wood TJ. 1965. Albino red squirrel collected in Wood Buffalo Park. The Blue Jay 23(2): 90.

Editor: Diego J. Lizcano

Recibido 2020-11-19

Revisado 2020-12-10

Aceptado 2021-02-12

Publicado 2021-04-03 